\title{
APROVEITAMENTO DE SUBPRODUTO INDUSTRIAL DE ÓlEOS VEGETAIS PARA PRODUÇÃO DE RIBOFLAVINA POR Candida guilliermondii DM $\mathbf{6 4 4}^{1}$
}

\author{
Maria de Lourdes Andrade PESSOA ${ }^{2, *}$, Samara Alvachian Cardoso ANDRADE ${ }^{3}$, \\ Alexandra Amorim SALGUEIRO ${ }^{3}$, Tânia Lúcia Montenegro STAMFORD²
}

\begin{abstract}
RESUMO
A produção e consumo de alimentos industrializados têm aumentado a preocupação com suplementação e enriquecimento de alimentos com vitaminas e sais minerais, visando repor as possíveis perdas durante os processos de fabricação, principalmente das vitaminas hidrossolúveis, mais especificamente da vitamina $\mathrm{B}_{2}$ ou riboflavina. Assim sendo, a proposta deste trabalho foi utilizar como componente principal do meio, para produção da riboflavina, um subproduto do refino de óleos vegetais e o microrganismo Candida guillermondii DM 644. A produção da vitamina $B_{2}$ foi realizada por fermentação em batelada utilizando Erlenmeyer. As condições empregadas foram agitação orbital, ausência de luz, $30^{\circ} \mathrm{C}$, e $24 \mathrm{~h}$ de incubação. A otimização da produção de riboflavina foi realizada através de Delineamento Fatorial Fracionário, para avaliar os efeitos da concentração de matéria graxa, fonte de nitrogênio, pH, velocidade de agitação, fonte de

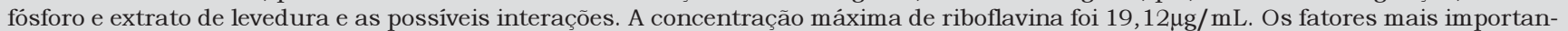
tes para produção de riboflavina foram a concentração de matéria graxa e a fonte de nitrogênio, enquanto que a fonte de fósforo e o extrato de leveduras não estimularam sua biossintese. A máxima produção foi obtida com matéria graxa a $10 \mathrm{~g} / \mathrm{L}, \mathrm{uréia}$ a $2,5 \mathrm{~g} / \mathrm{L}$ e $\mathrm{pH}$ 5,0. A velocidade de agitação (200 e 400rpm) não interferiu no processo biotecnológico.

Palavras-chave: vitamina $\mathrm{B}_{2}$; levedura; resíduo industrial de óleos vegetais.
\end{abstract}

\section{SUMMARY}

UTILIZATION INDUSTRIAL WASTE FROM VEGETAL OILS RIBOFLAVINE PRODUCTION BY Candida guilliermondii DM 644. The ever growing production and consumption of industrialized foods have increased the concern about supplementation and enrichment of food with vitamins and minerals, attempting the replenishment of the possible losses during their production processes, mainly the hydrosoluble vitamins, more specifically $\mathrm{B}_{2}$ vitamin or riboflavin. A subproduct of vegetal oil refining and the microorganism Candida guilliermondii DM 644 were used as the main substrate. The vitamin $\mathrm{B}_{2}$ was produced by the batch fermentation process using flasks with microorganism suspension, kept in orbital agitation, in the dark at $30^{\circ} \mathrm{C}$ for 24 hours. Optimization of the riboflavina production was carried out using a fractional factorial design, to evaluate the effects of oil substrate concentration, nitrogen source, pH, orbital agitation velocity, phosphate source, and yeast extract and the possible interactions. The maximum value of riboflavin concentration was $19.12 \mu \mathrm{g} / \mathrm{mL}$. The most important factors for riboflavin production were oil substrate concentration and nitrogen source, while phosphate source and yeast extract did not stimulate riboflavin production. The best conditions to produce riboflavin by C. guilliermond $i i$ DM 644 were: oil substrate $10 \mathrm{~g} / \mathrm{L}$, urea $2.5 \mathrm{~g} / \mathrm{L}$ and $\mathrm{pH}$ 5.0. The orbital agitation velocity (200 and 400rpm) did not interfere in the biotechnological process.

Keywords: vitamin $\mathrm{B}_{2}$; yeast; vegetable oil industrial waste.

\section{1 - INTRODUÇÃO}

A riboflavina ou vitamina $\mathrm{B}_{2}$ faz parte do complexo $\mathrm{B}$ cujo consumo mundial é de aproximadamente 1.250 toneladas por ano. De acordo com a fonte de onde é extraída, ela poderá ter várias denominações como: lactoflavina, ovoflavina, hepatoflavina e uroflavina [12]. Suas principais fontes na alimentação são carne, ovo, pescado e leite, apresentando ainda grandes variações de concentração entre as hortaliças e leguminosas. Devese ressaltar ainda a riqueza dessa vitamina nas leveduras. Sua forma livre é um produto natural relativamente raro, ocorrendo em maiores concentrações na retina, urina, leite e fluido seminal de animais, como também em alguns órgãos vegetais [19].

Essa vitamina é comercialmente produzida por sintese química e por fermentação microbiana, sendo esta última preferida, pois tem propiciado o desenvolvimento de novas tecnologias com diminuição de custos e alta

1. Recebido para publicação em 23/07/2002. Aceito para publicação em 27/01/2003 (000901).

2 Universidade Federal de Pernambuco, Centro de Ciências da Saúde, Departamento de Nutrição, Recife - PE,Brasil,newtonps@novaera.com.br

${ }^{3}$ Universidade Federal de Pernambuco, Departamento de Engenharia Química

* A quem a correspondência deve ser enviada. produtividade. Deve ser ressaltado que a riboflavina é a única vitamina que é rápida e totalmente sintetizada em altas concentrações por microrganismos.

Com o aumento do consumo de produtos alimentícios industrializados, aumenta a preocupação com a perda de nutrientes durante o processo de fabricação dos mesmos, principalmente no que diz respeito às perdas de vitaminas hidrossolúveis, essenciais ao bom funcionamento e desenvolvimento do organismo. A maior parte da riboflavina é usada em formulações farmacêuticas, enriquecimento de alimentos e suplementos alimentares para indústrias, de forma a minimizar essas perdas $[8,14]$.

Considerando os resultados promissores obtidos utilizando resíduos industriais na composição de meios de cultivo para produção de riboflavina por microrganismo, o aproveitamento de uma matéria bruta de baixo custo (subproduto ou resíduo industrial) é uma alternativa viável na investigação da produção dessa vitamina.

O presente trabalho teve como objetivo a produção de riboflavina por processo biotecnológico cultivando Candida guilliermondii DM644 num subproduto de refinaria de óleos vegetais, considerando que há uma carência a nivel nacional de produção dessa vitamina e que o mercado consumidor tende a se expandir. Por ou- 
tro lado, sua produção industrial pode ser economicamente viável pela utilização de um substrato de baixo custo.

\section{2 - MATERIAL E MÉTODOS}

\section{1 - Material}

\subsection{1 - Microrganismo utilizado e manutenção}

A linhagem de Candidaguilliermondii DM644 foi procedente da Micoteca do Departamento de Micologia da Universidade Federal de Pernambuco.

A cultura de C. guilliermondii foi mantida em tubos de ensaios com o meio de cultura descrito por OZBAS \& KUTSAL [13], com a seguinte composição em $\mathrm{gL}^{-1}: 20$ de glicose; 5 de peptona; 5 de extrato de levedura; 5 de extrato de malte; 0,2 de $\mathrm{MgSO}_{4} .7 \mathrm{H}_{2} \mathrm{O} ; 0,2$ de $\mathrm{K}_{2} \mathrm{HPO}_{4:} \mathrm{H}_{2} \mathrm{O}$ deionizada q.s.p. $1000 \mathrm{~mL}$ e ágar $2 \%$, este meio foi esterilizado em autoclave a $121^{\circ} \mathrm{C}$ por $20 \mathrm{~min}$.

\subsection{2 - Meio de fermentação e condições empre- gadas}

O subproduto industrial denominado de "borra", foi cedido pela SANBRA - Divisão da SANTISTA Alimentos $\mathrm{S} / \mathrm{A}$. Este subproduto, foi transferido de tanques industriais para depósitos de plásticos vedados, transportados para o laboratório em temperatura ambiente, onde foram armazenados em sacos plásticos com capacidade $200 \mathrm{~mL}$, congelados $\mathrm{a}-2{ }^{\circ} \mathrm{C}$ e descongelados apenas nos dias da preparação do meio de cultivo ou da determinação da sua composição físico-química.

Para a produção de riboflavina foi utilizado um meio liquido contendo como fonte de carbono a "borra" em concentrações diferentes, ajustado com solução de $\mathrm{HCl}$ para diferentes valores de $\mathrm{pH}$ e esterilizado a $121^{\circ} \mathrm{C}$ por 20 minutos; como fonte de nitrogênio foi utilizada a uréia ou $\left(\mathrm{NH}_{4}\right)_{2} \mathrm{SO}_{4}$, na proporção de nitrogênio:carbono de 1:4, $\mathrm{KH}_{2} \mathrm{PO}_{4}$ e extrato de levedura nas concentrações de $0,20 \mathrm{~g} / \mathrm{L}$ e $0,01 \mathrm{~g} / \mathrm{L}$ respectivamente, e agitação orbital de 200 ou 400 rpm por $24 \mathrm{~h}$, a temperatura de $30^{\circ} \mathrm{C}$ e no escuro, utilizando o processo de fermentação em batelada com frascos de Erlenmeyer de $500 \mathrm{~mL}$ contendo $150 \mathrm{~mL}$ do meio para produção, inoculado com suspensão de $C$. guillermondii. Essas variações estão especificadas no Delineamento Experimental.

\section{2 - Composição centesimal da "borra"}

Foram realizadas análises de umidade, cinzas, matéria graxa e proteínas em três lotes diferentes da "borra", armazenados sob congelamento, de acordo com a metodologia do INSTITUTO ADOLFO LUTZ [7]; o teor de metais foi determinado por espectrometria de absorção atômica no Laboratório de Análises de Minerais da SUDENE.

\section{3 - Padronização do inóculo}

Foi preparada uma densa suspensão de células a partir de culturas de C. guilliermondii. Os pré-inóculos foram preparados em frascos de Erlenmeyer de $50 \mathrm{~mL}$ contendo $15 \mathrm{~mL}$ do meio liquido ("borra”), para produção de riboflavina, incubados por $12 \mathrm{~h}$ sob agitação orbital de 200 ou $400 \mathrm{rpm}$, à temperatura de $30^{\circ} \mathrm{C}$ na ausência de luz. Foi utilizado $10 \%$ do inóculo.

\section{4 - Produção de riboflavina}

Os experimentos para otimização da produção de riboflavina foram realizados utilizando frascos de Erlenmeyer de $500 \mathrm{~mL}$ de capacidade, contendo $150 \mathrm{~mL}$ do meio liquido ("borra"), cujas condições foram investigadas de acordo com o planejamento experimental, sendo constantes os inóculos. Para a extração e quantificação da riboflavina foram coletadas aliquotas em triplicata de 5,0mL.

\section{5 - Delineamento experimental}

Para otimização das condições de produção de riboflavina por Candida guilliermondii foram utilizados dois planejamentos fatoriais fracionários $2^{4-1}$ [2]. As variáveis independentes para o primeiro planejamento fatorial fracionário foram: 1 - concentração da "borra"; 2 - fonte de nitrogênio; 3 - pH; 4 - agitação orbital. Para o segundo planejamento fatorial fracionário foram: concentração da "borra"; $\mathrm{pH} ; \mathrm{KH}_{2} \mathrm{PO}_{4}$; extrato de levedura. A resposta de interesse para os dois planejamentos foi à produção de riboflavina por C. guilliermondii. Os niveis decodificados de cada variável são apresentados nas Tabelas 1 e 2. Cada planejamento consistiu de 8 ensaios, cujas condições (niveis codificados) são apresentadas nas Tabelas 3 e 4 .

TABELA 1. Níveis decodificados das variáveis do primeiro planejamento fatorial.

\begin{tabular}{ccccc}
\hline Niveis & \multicolumn{4}{c}{ Niveis decodificados } \\
\cline { 2 - 5 } codificados & $\begin{array}{c}\text { Concentração da } \\
\text { "borra" }(\mathrm{g} / \mathrm{L})\end{array}$ & $\begin{array}{c}\text { Fonte de } \\
\text { nitrogênio }\end{array}$ & $\mathrm{pH}$ & $\begin{array}{c}\text { Agitação orbital } \\
(\mathrm{rpm})\end{array}$ \\
\hline-1 & 5 & $\left(\mathrm{NH}_{4}\right)_{2} \mathrm{SO}_{4}$ & 4,0 & 200 \\
1 & 10 & Uréia & 7,0 & 400 \\
\hline
\end{tabular}

TABELA 2. Níveis decodificados das variáveis do segundo planejamento fatorial.

\begin{tabular}{|c|c|c|c|c|}
\hline \multirow{2}{*}{$\begin{array}{c}\text { Níveis } \\
\text { codificados }\end{array}$} & \multicolumn{4}{|c|}{ Níveis decodificados } \\
\hline & $\begin{array}{l}\text { Concentração da } \\
\text { "borra" (g/L) }\end{array}$ & $\mathrm{pH}$ & $\mathrm{KH}_{2} \mathrm{PO}_{4}(\mathrm{~g} / \mathrm{L})$ & $\begin{array}{c}\text { Extrato de } \\
\text { levedura }(g / L)\end{array}$ \\
\hline-1 & 7,5 & 3,0 & ausência & ausência \\
\hline 1 & 10 & 5,0 & 0,20 & 0,01 \\
\hline
\end{tabular}

TABELA 3. Níveis codificados das variáveis para os ensaios do primeiro planejamento fatorial.

\begin{tabular}{ccccc}
\hline Ensaios & $\begin{array}{c}\text { Concentração } \\
\text { da "borra" }\end{array}$ & $\begin{array}{c}\text { Fonte de } \\
\text { Nitrogênio }\end{array}$ & $\mathrm{pH}$ & $\begin{array}{c}\text { Agitação } \\
\text { orbital }\end{array}$ \\
\hline 1 & $1-$ & $1-$ & $1-$ & $1-$ \\
2 & $1+$ & $1-$ & $1-$ & $1+$ \\
3 & $1-$ & $1+$ & $1-$ & $1+$ \\
4 & $1+$ & $1+$ & $1-$ & $1-$ \\
5 & $1-$ & $1-$ & $1+$ & $1+$ \\
6 & $1+$ & $1-$ & $1+$ & $1-$ \\
7 & $1-$ & $1+$ & $1+$ & $1-$ \\
8 & $1+$ & $1+$ & $1+$ & $1+$ \\
\hline
\end{tabular}


TABELA 4. Níveis codificados das variáveis para os ensaios do segundo planejamento fatorial.

\begin{tabular}{ccccc}
\hline Ensaios & $\begin{array}{c}\text { Concentração } \\
\text { da "borra" }\end{array}$ & $\mathrm{pH}$ & $\mathrm{KH}_{2} \mathrm{PO}_{4}$ & $\begin{array}{c}\text { Extrato de } \\
\text { levedura }\end{array}$ \\
\hline 1 & $1-$ & $1-$ & $1-$ & $1-$ \\
2 & $1+$ & $1-$ & $1-$ & $1+$ \\
3 & $1-$ & $1+$ & $1-$ & $1+$ \\
4 & $1+$ & + & $1-$ & $1-$ \\
5 & $1-$ & $1-$ & $1+$ & $1+$ \\
6 & $1+$ & $1-$ & $1+$ & $1-$ \\
7 & $1-$ & $1+$ & $1+$ & $1-$ \\
8 & $1+$ & $1+$ & $1+$ & $1+$ \\
\hline
\end{tabular}

Para quatro fatores a serem investigados num planejamento experimental, o número de experimentos a serem realizados no planejamento fatorial completo é 16 (dezesseis). Considerando uma meia fração desse planejamento, teremos o planejamento fatorial fracionário $2^{4-1}$ sendo necessário apenas 8 (oito) experimentos. O primeiro planejamento fatorial foi realizado em triplicata e o segundo em duplicata.

\section{6 - Determinação de pH}

$\mathrm{O}$ pH das amostras coletadas foi medido através de potenciometria ao longo da fermentação para produção de riboflavina.

\section{7 - Determinação de riboflavina}

A extração dessa vitamina foi realizada segundo o método descrito por GOODWIN \& PENDLINGTON [6], modificado, de acordo com a Figura 1.
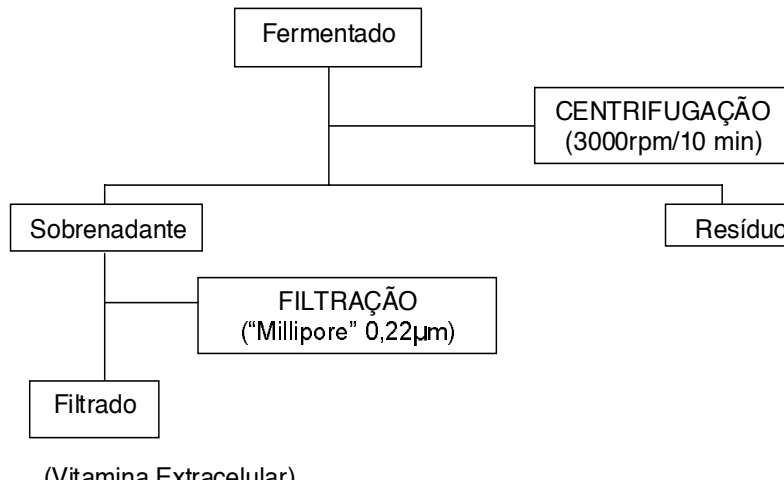

FIGURA 1. Esquema de extração de riboflavina extracelular.

Aliquotas do cultivo de C. guilliermondii DM 644 foram coletadas assepticamente e armazenadas no escuro até serem centrifugadas a 3000 rpm por $10 \mathrm{~min}$ para separação da massa celular do sobrenadante. Após centrifugação, foi realizada filtração em membrana "Millipore" de $0,22 \mu \mathrm{m}$, visando retirar o excesso de matéria-graxa. No filtrado livre de células e matéria graxa foi determinado o conteúdo de riboflavina extracelular, cobrindo sempre as amostras com papel laminado para evitar a degradação dessa vitamina pela luz.
Para quantificação da riboflavina foi utilizado o método direto segundo a técnica descrita por GOODWIN \& PENDLINGTON [6] e SABRY, EL-REFAI \& GAMATI [17].

A partir do filtrado de cada amostra convenientemente manipulada, foi determinado o conteúdo de vitamina extracelular pela leitura de absorbância a $445 \mathrm{~nm}$ e por intermédio de uma regressão linear do padrão de riboflavina nas concentrações de 0,1 a $50 \mu \mathrm{g} / \mathrm{mL}$.

As leituras de absorbância foram feitas contra um branco constituído pelo meio de cultura para a produção de riboflavina filtrado também por membrana

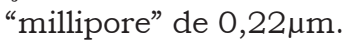

\section{8 - Métodos estatísticos}

As respostas obtidas para todos os ensaios foram avaliadas quanto aos efeitos principais e as interações entre os fatores, conforme a metodologia descrita por BARROS NETO, SCARMINIO \& BRUNS [2], obtidas através do programa Statistica versão 6.0.

\section{3 - RESULTADOS E DISCUSSÃO}

A composição físico-química da "borra" está representada na Tabela 5.

TABELA 5. Composição centesimal da "borra".

\begin{tabular}{lc}
\hline Componentes & $\%^{\star}$ \\
\hline Umidade & 9,82 \\
Matéria graxa & 46,46 \\
Carboidratos $^{\star \star}$ & 34,91 \\
Proteínas & 0,55 \\
Cinzas & 8,26 \\
\hline
\end{tabular}

* Peso úmido
** Calculados por diferença

A levedura utilizou esse subproduto como fonte de carbono devido ao seu elevado percentual de carboidratos e matéria graxa (46\%). Resultados promissores têm sido obtidos na produção dessa vitamina pela mesma levedura, utilizando como fontes de carbono fração da refinação de óleos [16]; óleo de babaçu [12].

A concentração de metais do subproduto industrial encontra-se na Tabela 6.

TABELA 6. Concentração de metais na "borra"

\begin{tabular}{lc}
\hline \multicolumn{1}{c}{ Metal } & $\%^{*}$ \\
\hline Ferro $\left(\mathrm{em} \mathrm{Fe}^{3+}\right)$ & $\mathrm{ND}$ \\
Cobalto $\left(\mathrm{Co}^{2+}\right)$ & $\mathrm{ND}$ \\
Sódio $\left(\mathrm{em} \mathrm{Na}^{+}\right)$ & 0,7000 \\
Cálcio $\left(\mathrm{em} \mathrm{Ca}^{2+}\right)$ & 0,0800 \\
Magnésio $\left(\mathrm{em} \mathrm{Mg}^{2+}\right)$ & 0,0630 \\
Potássio $\left(\mathrm{em} \mathrm{K}^{+}\right)$ & 0,0050 \\
Cobre $\left(\mathrm{em} \mathrm{Cu}^{2+}\right)$ & 0,0011 \\
Manganês $\left(\mathrm{em} \mathrm{Mn}^{2+}\right)$ & 0,0041 \\
Zinco $\left(\mathrm{em} \mathrm{Zn}^{2+}\right)$ &
\end{tabular}

* Peso úmido

$\mathrm{ND}=$ não detectável 
A ausência de íon $\mathrm{Fe}^{3+}$ na "borra" pode ser determinante no aproveitamento desse subproduto industrial para produção de riboflavina. Vários trabalhos já foram publicados ressaltando o efeito negativo desse ín na produção dessa vitamina [4,5,21].

Por outro lado, SABRY \& GHOZLAN [18], observaram que a produção de riboflavina não foi influenciada pelos metais presentes no melaço quando cultivaram Aspergillus terreus na presença de resíduo da fabricação de milho como fonte de nitrogênio.

A presença dos íons $\mathrm{Na}^{+}, \mathrm{K}^{+}, \mathrm{Ca}^{2+}, \mathrm{Mg}^{2+}, \mathrm{Mn}^{2+}, \mathrm{Zn}^{2+} \mathrm{e}$ $\mathrm{Cu}^{2+}$ em concentrações minimas, no subproduto industrial utilizado nesse trabalho, tende a contribuir positivamente no metabolismo do microrganismo devido a esses ions constituírem-se em cofatores de inúmeras enzimas [1].

YAMANE, OOSHIMA \& KATO [21], evidenciaram o efeito positivo do íon $\mathrm{Zn}^{2+}$ na produção de riboflavina por Arthrobacter na presença dos íons $\mathrm{K}^{+}, \mathrm{Mg}^{2+}$ e $\mathrm{Ca}^{2+}$.

Pode-se observar na Tabela 7 que a melhor produtividade média de riboflavina foi de $18,22 \mu \mathrm{g} / \mathrm{mL}$. dia, obtida quando a fonte de nitrogênio e carbono estão em seus niveis superiores. Comparando os dois ensaios $(4 \mathrm{e}$ 8), podemos observar que apresentam os mesmos niveis da fonte de carbono e nitrogênio, só diferem pelo nivel do $\mathrm{pH}$ e da agitação orbital. O ensaio correspondendo ao nivel superior destes fatores independentes teve uma queda da produtividade média da riboflavina. Essa diferença entre eles foi muito pequena, menor que $16 \%$. Conseqüentemente, as faixas de $\mathrm{pH}$ e de agitação do cultivo de $C$. guilliermondii não devem interferir na produção de riboflavina, discordando dos achados de BAILEY $\&$ OLLIS [1], que nos cultivos submersos de microrganismos, observaram que a agitação influenciou diretamente a aeração por aumentar a quantidade de oxigênio dissolvido no meio de cultura, oferecendo uma maior superficie de contato entre o gás e as células e facilitando a transferência de massa nos processos aeróbios.

TABELA 7. Produtividade de riboflavina por C. guilliermondii DM 644 em três séries de experimentos para o primeiro planejamento fatorial fracionário.

\begin{tabular}{ccccccccc}
\hline \multicolumn{1}{c}{ Produtividade $(\mu \mathrm{g} / \mathrm{mL}$.dia $)$} \\
\hline Ensaio & A & B & C & D & $1^{\text {a }}$ série & $2^{\text {a }}$ série & $3^{\text {a }}$ série & Média \\
1 & - & - & - & - & ND & 1,72 & ND & 0,57 \\
2 & + & - & - & + & 10,85 & ND & 1,53 & 4,13 \\
3 & - & + & - & + & ND & 7,91 & 10,97 & 6,29 \\
4 & + & + & - & - & 1,03 & 44,42 & 9,20 & 18,22 \\
5 & - & - & + & + & ND & ND & 0,91 & 0,30 \\
6 & + & - & + & - & ND & ND & 6,54 & 2,18 \\
7 & - & + & + & - & 16,81 & ND & ND & 5,60 \\
8 & + & + & + & + & 0,39 & 41,96 & 3,78 & 15,38 \\
\hline
\end{tabular}

ND - não detectado; A-concentração da"borra"(g/L); B-fonte de nitrogênio; C-pH inicial; D-agitação orbital(rpm).

A importância da concentração da "borra" pode ser vista na Tabela 7. Quanto maior a concentração de ma- téria graxa no meio de cultivo de C. guilliermondii, maior a produção de riboflavina, resultados estes concordantes aos encontrados por KALINGAN \& KRISHNAN [9], quando investigaram o efeito da concentração de melaço como fonte de carbono na produção de riboflavina por E. ashbyii, detectando o aumento gradativo da vitamina à medida que aumentava a concentração do melaço na faixa de 3 a 5\%. Logo, a concentração inicial da matéria graxa foi novamente investigada no segundo planejamento fatorial.

Os resultados apresentados na Tabela 8 mostram que os efeitos concentração da "borra" e fonte de nitrogênio são os mais importantes, embora não tenham exercido influência significativa nas respostas, dentro das faixas estudadas.Quanto ao fator $\mathrm{pH}$, (Tabela 8), embora não significativo, houve uma pequena tendência da produção dessa vitamina ser estimulada em $\mathrm{pH}$ ácido (Tabela 7). Estes resultados são confirmados por KOLONNE, SEVIOUR \& MCDOUGALL [10] no cultivo de $E$. ashbyii na presença de glicose e em $\mathrm{pH}$ ao redor de 4,5-5,5, enquanto nenhuma produção de riboflavina foi detectada a $\mathrm{pH} 3,5$ e 8,5. Portanto, foi investigado no segundo planejamento fatorial os valores de $\mathrm{pH} 3,0$ e 5,0 .

TABELA 8. Efeitos principais e de interação para o primeiro planejamento fatorial fracionário.

\begin{tabular}{ccc}
\hline Fatores e Interações & Efeitos Principais & Efeitos de Interação \\
\hline$A$ & 6,8 & - \\
$B$ & 9,6 & - \\
$C$ & $-1,4$ & - \\
$D$ & $-0,1$ & - \\
$A B=C D$ & - & 4,1 \\
$A C=B D$ & - & $-1,0$ \\
$A D=B C$ & - & $-0,3$ \\
$A B C=D$ & & $-0,1$ \\
\hline
\end{tabular}

A - concentração da "borra"(g/L); B - fonte de nitrogênio; C - pH; D - agitação orbital (rpm).

Para o fator agitação, os resultados obtidos demonstraram que a variação de 200 para 400rpm não foi significativa (Tabela 8). Logo, no segundo planejamento fatorial, foi usado 400rpm, conseguindo-se assim uma melhor dispersão do substrato em toda a extensão do cultivo. Segundo OZBAL \& KUTSAL [14], e YAMANE et al. [22], que trabalharam com o cultivo de A. gosypii e Arthrobacter sp. , os melhores resultados para a produção de riboflavina foram obtidos sob agitação de 300rpm e 600rpm, respectivamente.

Analisando os efeitos de interação entre dois fatores na Tabela 8, foi observada a não significância ao nivel de $5 \%$ de todos eles. Entretanto, houve uma importante interação entre a matéria graxa e fonte de nitrogênio, podendo-se afirmar que uma variação do fator fonte de carbono é dependente da fonte de nitrogênio e esses dois fatores não devem ser analisados separadamente. Apesar do efeito de interação entre os fatores pH e agitação ter apresentado o valor 4,1 , pode-se observar que seus efeitos principais foram baixos. 
Os resultados apresentados na Figura 2 mostram que, com relação ao sal de amônio, a produtividade mé-

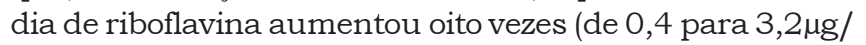
$\mathrm{mL}$ dia), e, na presença de uréia, aumentou de 5,9 para $17 \mu \mathrm{g} / \mathrm{mL}$ dia, ou seja 3 vezes. Pode-se também observar que a fonte de nitrogênio orgânica estimula a produção de riboflavina dez vezes mais que o sal de amônio quando a concentração de "borra" é $5 \mathrm{~g} / \mathrm{L}$. Comportamento metabólico diferente é evidenciado quando essa fonte de carbono é de $10 \mathrm{~g} / \mathrm{L}$. Possivelmente há inibição por excesso de substrato. Os resultados acima discordam dos achados por LEVINE et al. [11], que constataram aumento na produção de riboflavina por C. flareri na presença de sulfato de amônio, enquanto a uréia causou efeito inverso no metabolismo celular dessa levedura. Diante destes resultados, foi utilizada na segunda etapa do planejamento experimental a fonte de nitrogênio orgânica (uréia), sempre mantendo-se a taxa: fonte de nitrogênio: fonte de carbono igual a $1: 4$.

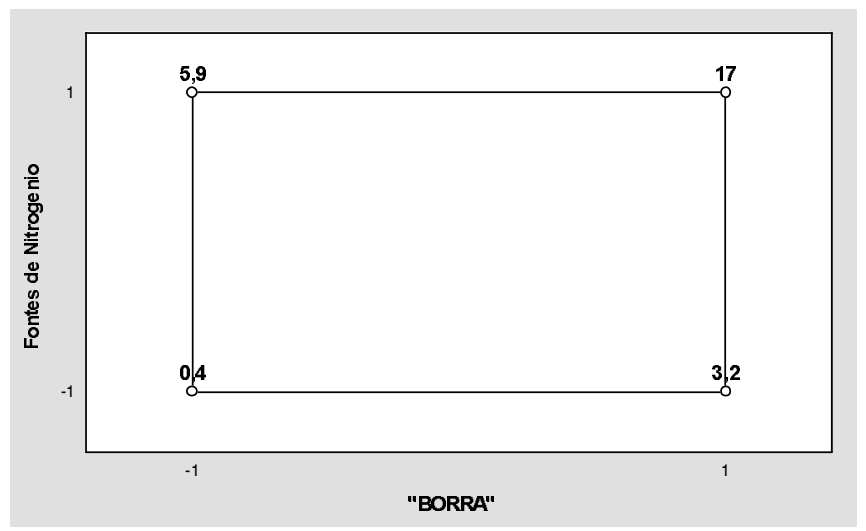

FIGURA 2. Interpretação dos resultados de produtividade média do primeiro planejamento fatorial fracionário.

O melhor resultado médio de riboflavina produzido por C. guilliermondii foi obtido com pH 5,0; concentração

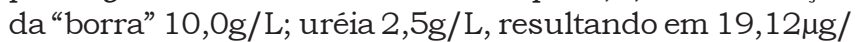
mL.dia (Tabela 9).

Ao calcular a resposta dos ensaios no segundo planejamento fatorial, observa-se na Tabela 10 que a variável independente $\mathrm{pH}$ foi a única a exercer influência significativa sobre a produção de riboflavina.

A elevação da concentração da "borra" acarretou um aumento da produtividade, que foi mais acentuada em pH 5,0 $(0,21$ para 10,77) (Figura 3).

Com relação ao fósforo, ficou evidenciado que ele não estimulou a produção (Tabelas 9 e 10), reforçando o que foi dito por SCHLEGEL [20], que possivelmente a mesma velocidade de agitação e conseqüentemente a mesma condição de aeração utilizada na investigação dos dois niveis do fator independente fósforo justificam os resultados obtidos, considerando que para maior concentração de fósforo no meio é necessário uma maior concentração de oxigênio para que ocorra liberação de ATP por fosforilação oxidativa.
TABELA 9. Produtividade de riboflavina por C. guilliermondii DM 644 em três séries de experimentos para o segundo planejamento fatorial fracionário.

\begin{tabular}{cccccccc}
\hline Ensaio & $\mathrm{E}$ & $\mathrm{F}$ & $\mathrm{G}$ & $\mathrm{H}$ & $1^{\mathrm{a}}$ série & $2^{\mathrm{a}}$ série & Média \\
\hline 1 & - & - & - & - & $\mathrm{ND}$ & 0,09 & 0,04 \\
2 & + & - & - & + & 0,91 & 0,99 & 0,95 \\
3 & - & + & - & + & 5,77 & 6,56 & 6,16 \\
4 & + & + & - & - & 30,48 & 7,76 & 19,12 \\
5 & - & - & + & + & $\mathrm{ND}$ & 0,98 & 0,49 \\
6 & + & - & + & - & $\mathrm{ND}$ & $\mathrm{ND}$ & $\mathrm{ND}$ \\
7 & - & + & + & - & 0,67 & $\mathrm{ND}$ & 0,34 \\
8 & + & + & + & + & 14,00 & 3,45 & 8,92 \\
\hline
\end{tabular}

ND: não detectado; E-matéria graxa (g/L); F:pH; G: $\mathrm{KH}_{2} \mathrm{PO}_{4}(\mathrm{~g} / \mathrm{L})$; H: Extrato de levedura $(\mathrm{g} / \mathrm{L})$.

TABELA 10. Efeitos principais e de interação para o segundo planejamento fatorial fracionário.

\begin{tabular}{ccc}
\hline Fatores e Interações & Efeitos Principais & Efeitos de Interação \\
\hline$E$ & 5,44 & - \\
$F$ & $8,21^{*}$ & - \\
$G$ & $-4,18$ & - \\
$H$ & $-0,79$ & - \\
$E F=G H$ & - & 5,23 \\
$E G=F H$ & - & $-1,49$ \\
$E H=F G$ & - & $-3,93$ \\
$E F G=H$ & & $-0,79$
\end{tabular}

*Efeito significativo; E: matéria graxa (g/L); F: pH; G- $\mathrm{KH}_{2} \mathrm{PO}_{4}$ (g/L); H: Extrato de levedura $(\mathrm{g} / \mathrm{L})$

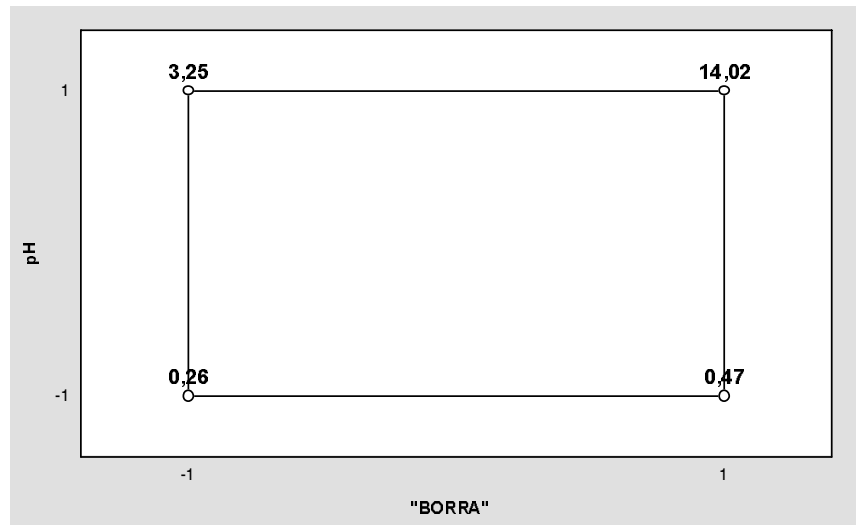

FIGURA 3. Interpretação dos resultados de produtividade do segundo planejamento fatorial fracionário.

Com relação ao extrato de levedura (Tabela 10), fica evidenciado que a presença de vitaminas do complexo $\mathrm{B}$, presentes no extrato, poderiam atuar como precursores da riboflavina ou mesmo, como fator de crescimento para C. guilliermondii, porém não influenciaram a produção de riboflavina nas condições de trabalho, o que pode ser explicado por BLACKWELL, SINGLETON \& TOBIN [3], que afirmaram que possivelmente os cultivos de C. guilliermondii não são estimulados pelo extrato de levedura devido a efeitos antagônicos entre os metais presentes na "borra" e os componentes dessa variável investigada. Em contraste, o extrato de levedura é metabolisado por microrganismos não apenas como fonte 
de nitrogênio, mas também como fonte de carbono, vitaminas, aminoácidos e sais minerais [15].

\section{4 - CONCLUSÕES}

Os resultados obtidos no presente trabalho permitem inferir as seguintes conclusões:

- No primeiro planejamento fatorial fracionário, a produção de riboflavina foi influenciada pelas variáveis independentes (fonte de carbono e nitrogênio) e suas interações, embora não tenha dado diferença significativa;

- Para a produção de riboflavina, as condições ótimas de cultivo foram: matéria graxa $10 \mathrm{~g} / \mathrm{L}$, uréia $2,5 \mathrm{~g} / \mathrm{L}$ e $\mathrm{pH} 5,0$, não interferindo a velocidade de agitação (200 e 400rpm);

- $\mathrm{O}$ extrato de levedura e fonte de fósforo $\left(\mathrm{KH}_{2} \mathrm{PO}_{4}\right)$ não estimularam a produção de riboflavina;

- A produção de riboflavina foi significativa com $\mathrm{pH}$ 5,0 , tido maior produtividade com a concentração da "borra" $10 \mathrm{~g} / \mathrm{L}$;

- A produtividade máxima de riboflavina obtida no planejamento fatorial 1 e 2 situou-se entre 18 $20 \mu \mathrm{g} / \mathrm{mL}$.dia.

\section{5 - REFERÊNCIAS BIBLIOGRÁFICAS}

[1] BAILEY, J.E.; OLLIS, D.F. Biochemical Engineering Fundamentals. Singapure: McGraw-Hill, 2a ed, 984p, 1986.

[2] BARROS NETO, B.; SCARMINIO,I.S.; BRUNS, R. E. Planejamento e Otimização de experimentos, Campinas, SP: Editora da UNICAMP, 299p, 1995.

[3] BLACKWELL, K. J.; SINGLETON, I. \& TOBIN, J. M. Metal cation uptake by yeast: a review. Appl. Microbiol Biotechnol., v. 43, p. 579-584, 1995.

[4] ENARI, T. M.; KAUPPINEN, V. Interaction of cobalt and iron in the riboflavin production of Candida guilliermondii. Acta Chemica Sacandinavia, v. 15, n. 7, p. 1513-1516, 1961.

[5] GHOZLAN, H. A. Utilization of beet molasses for riboflavin production by Mycobacterium phlei. J. Basic. Microbiol., v. 34, n. 3, p. 157-162, 1994.

[6] GoOdwin, T. W.; PEndlington, s. Studies on the Biosynthesis of Riboflavin nitrogen metabolism and flavinogeneses in Eremothecium ashbyii. Biochemistry Journal, v. 57, p. 631-641, 1954.

[7] INSTITUTO ADOLFO LUTZ. Métodos Químicos e Físicos para análise de Alimentos. In: Normas Analíticas do Instituto Adolfo Lutz, v. 1. São Paulo: Autor, $3^{\text {a }}$ ed., 1985.

[8] KALINGAN, A. E.; KRISHNAN, M. R. V. Aplication of agroindustrial by -products for riboflavin production by
Eremothecium ashbyii NRRL 1363. Appl. Microbiol. Biotechnol, v. 47, p. 226-230, 1997a.

[9] KALINGAN, A. E.; KRISHNAN, M. R. V. Agro industrial byproducts as flavinogenic stimulators for riboflavin production. Bioprocess Engineering, v. 17, p. 8791,1997b.

[10] KOLONNE, S.; SEVIOUR, R.J. \& McDOUGALL, B.M. Effect of $\mathrm{pH}$ on exocellular riboflavin production by Eremothecium ashbyii. Biotechnology Letters, v. 16, n. 1, p. 79-84, 1995 .

[11] LEVINE, H.; OYAAS, J. E.; WASSERMAN, L.; HOOGERHEIDE, J. C. \& STERN, R. M. Riboflavin production by Candida Yeast. Industrial and Engineering Chemistry, v. 41, n. 8, p. 1661-1668, 1949.

[12] NEVES, M. L. C. Produção de riboflavina por Candida guilliermondii (Cast.) Lang e Guerra utilizando óleos de girassol, soja e babaçu. Recife, 1994. Dissertação de Mestrado, Centro de Ciências da Saúde, Universidade Federal de Pernambuco (UFPE).

[13] OZBAL, T. \& KUTSAL, T. Comparative study of riboflavin production from two microorganisms: Eremothecium ashbyii and Ashbyagossypii. Enzyme Microb. Technol., v. 8, p. 593-597, 1986.

[14] OZBAL, T.; KUTSAL, T. Effects of agitation and aeration rates on riboflavin fermentations by Ashbya gossypii. Biottechnology and Applied Biochemistry, v. 37, p. 97-105, 1991.

[15] ROSE, A. H. Responses to the chemical environment; in: The Yeast, v. 2; eds. A. H. Rose \& J.S. Harrison; Academis Press, London, p. 5- 40, 1987.

[16] SABRY, S.A.; EL-REFAI, A. H.; GAMATI, S.Y.Utilization of oil fraction (solar) for riboflavin production by Candida guilliermondii as influenced by some culture conditions. Biotechnology Letters, v. 10, n. 9, p. 615- 618, 1988.

[17] SABRY, S. A.; EL-REFAI, A. H.; GAMATI, S. Y. Production of riboflavin (Vitamin $\mathrm{B}_{2}$ ) by hydrocarbon-utilizing yeasts. Microbiologia Sem, v. 5, p. 45- 52, 1989.

[18] SABRY, S. A.; GHOZLAN, H. A. Effect of some nutrients on riboflavin by Aspergillus terreus. Rev. Lat.-Amer. Microbiol. v. 36, p. 27-32, 1994.

[19] SCHLEE, D.; STRAUGE, G. Physiology and biochemistry of riboflavin formation. Die Pharmazie, v. 39, p. 805-811, 1984.

[20]SCHLEGEL, H.G. Basic mechanism and energy conversion; in: GeneralMicrobiology; Reino Unido: Cambridge University Press, $7^{\text {th }}$ edição, p. 234-289, 1993.

[21] YAMANE, Y.; OOSHIMA, H.; KATO, J. Overproduction of riboflavin by an Arthrobacter sp. Mutant resistant to 5fluorouraci. Enzyme Microb. Technol., v. 15, p. 877880, 1993.

[22]YAMANE, Y.; NAKAMURA, Y.; OKAMOTO, H.; OOSHIMA, H.; KATO, J. Overpro-duction of riboflavin by an Arthrobacter sp. Mutant resistant to 5 - fluorouracil Effects of $\mathrm{pH}$ and dissolved oxygen concentration on production of riboflavin. Applied Biochemistry and Biotechnology, v. 50, p. 317-322, 1995. 